\title{
Novel multi-photon microscopy based on resonant nonlinear optics of colloidal quantum dots
}

\author{
Francesco Masia ${ }^{1, *}$, Wolfgang Langbein ${ }^{1}$, and Paola Borri ${ }^{2}$ \\ ${ }^{1}$ School of Physics and Astronomy, Cardiff University, The Parade, Cardiff CF24 3AA, United Kingdom \\ ${ }^{2}$ School of Biosciences, Cardiff University, Museum Avenue, Cardiff CF10 3AT, United Kingdom
}

Received 31 May 2008, accepted 30 October 2008

Published online 24 December 2008

PACS 78.47.Fg, 78.47.nj, 78.67.Hc

* Corresponding author: e-mail masiaf@cf.ac.uk, Phone: +44-29-20870167, Fax: +44-29-20874056

We demonstrate a novel imaging modality based on the detection of resonant four-wave mixing emitted by colloidal quantum dots. This third-order signal is excited and detected at a visible wavelength in resonance with an electronic transition, and results in a significant improvement of the spatial resolution with respect to a twophoton fluorescence microscope.
In particular, we have investigated the dependence of the transient reduced absorption of the sample induced by the illumination (photobleaching) on the exposure time and excitation intensity, and its influence to the fourwave mixing signal.
1 Introduction Optical microscopy is a key tool in cell biology and continuous effort is devoted to develop new techniques for in vitro and in vivo cell and tissue imaging with improved spatial and temporal resolution by exploiting new contrast agents and imaging mechanisms [1]. In recent years, semiconductor nanocrystals have attracted increasing attention as innovative inorganic probes able to overcome typical limitations of organic fluorophores for application in fluorescence microscopy [2-5]. Indeed, these nanocrystals also called colloidal quantum dots (CQDs) show wider absorption and narrower emission spectra compared to organic dyes and also superior brightness and photostability [6,7].

Among the various optical techniques reported, multiphoton microscopy based on nonlinear optics is especially interesting since it allows for an intrinsic three-dimensional (3D) resolution. For example, in a two-photon fluorescence (TPF) microscope the nonlinear absorption can take place mainly in the focal volume, allowing for optical sectioning without the need of a detector pinhole to reject out-of focus light, and in turn it also results in a reduced overall fluorophore photobleaching limited to a very small region of the sample [8].

CQDs show a large nonlinear absorption cross section and have been successfully exploited in TPF microscopy [9]. They also exhibit a large third-order optical nonlinearity and a four-wave mixing (FWM) signal can be generated by exciting them in resonance with the excitonic transitions [10]. In this work, we demonstrate that four-wave mixing from CQDs can be used as a novel imaging modality for microscopy with high spatial resolution and sensitivity. Besides retaining the inherent 3D resolution capability of multi-photon fluorescence, this technique benefits from a coherent detection, free from any incoherent background. Moreover, since it exploits a third-order nonlinearity in resonance with the excitonic transition (in the visible range for the most commonly available $\mathrm{CdSe} / \mathrm{ZnS} \mathrm{CQDs}$ ), the spatial resolution is significantly increased compared to TPF microscopy which is less nonlinear (quadratic) in the excitation intensity and uses the excitation light at twice the wavelength for the same detection wavelength. 


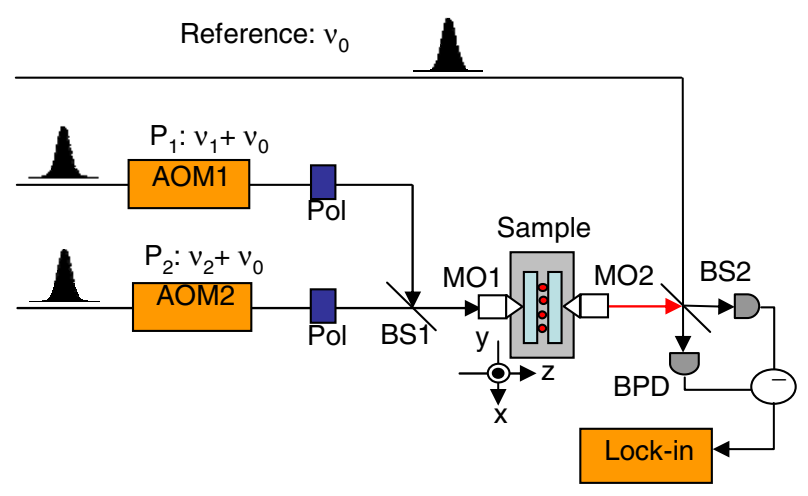

Figure 1 Sketch of the experimental set-up. AOM: Acoustooptic modulator. Pol: Polarization control via $\lambda / 2$ and $\lambda / 4$ waveplates. MO: Microscope objective. xyz: Piezoelectric scanning stage. BS: Unpolarized 50:50 beam splitters. BPD: Balanced photodiode.

2 Experiment Commercial trioctylphosphine oxide (TOPO) capped $\mathrm{CdSe} / \mathrm{ZnS}$ colloidal quantum dots (from Plasmachem $\mathrm{GmbH}$ ) were dispersed in a polymethylmethacrylate (PMMA)/toluene solution (final CQDs concentration $10 \mu \mathrm{M})$ and spin coated on a $0.17 \mathrm{~mm}$ thick glass coverslip. The photoluminescence (PL) spectrum at room temperature is centered at $610 \mathrm{~nm}$ with a linewidth full width at half maximum (FWHM) of $33 \mathrm{~nm}$, and we measured a PL quantum yield [11] of 40\%. In the FWM experiment, the CQDs are excited by Fourier-limited laser pulses of $150 \mathrm{fs}$ duration ( $4 \mathrm{~nm}$ spectral width) at $\nu_{0}=76 \mathrm{MHz}$ repetition rate. The pulse train is divided into three beams called reference, $P_{1}$, and $P_{2}$ (see Fig. 1). Two acoustooptic modulators (AOMs) are used to upshift their optical frequencies by $\nu_{1}=80 \mathrm{MHz}$ and $\nu_{2}=79 \mathrm{MHz}$, respectively. The time delay between $P_{1}$ and $P_{2}$ is controlled by a delay stage prior recombining them into the same spatial mode by the beam splitter BS1. $P_{1,2}$ are focused onto the sample by an oil-immersion microscope objective (MO1) with 1.25 numerical aperture (NA) to achieve high spatial resolution. The sample is moved with respect to the focal point of the objectives by a $x y z$ piezoelectric scanning unit with nanometric resolution. The generated FWM signal and the transmitted fields of $P_{1,2}$ are collected by a second objective (MO2) and interfere with the reference beam in the beam splitter BS2. The interference is detected by two balanced photodiodes (BPD) and a lock-in amplifier in a heterodyne scheme [12]. The transmitted $P_{1}$ and $P_{2}$ beams and the FWM signal can be distinguished by selecting the proper beating frequency $\left(\left(\nu_{1(2)}-\nu_{0}\right)=4(3) \mathrm{MHz}\right.$, for $P_{1}$ and $P_{2}$, respectively, and $\left(2 \nu_{2}-\nu_{1}-\nu_{0}\right)=2 \mathrm{MHz}$ for FWM). Since the FWM signal is detected interferometrically, it is free from any incoherent (e.g. fluorescence, Raman) background. An additional cross-polarization scheme is used to reject background due nonlinearities in the photodiodes. Two pairs of $\lambda / 4$ and $\lambda / 2$ waveplates determine the polarization state of the $P_{1,2}$ beams. $P_{1}$ is cross-polarized with respect to the reference beam to minimize the interference at the photodiodes, while the polarization of $P_{2}$ is chosen to maximize the detected FWM signal. For all the data reported in this work, the FWM signal is measured when the pump and probe pulses are in time overlap. In fact, we measured a homogeneous linewidth of the fundamental excitonic transition in the CQDs of $\sim 23 \mathrm{~nm}$ at room temperature from single dot luminescence which corresponds to a dephasing time of $\sim 20 \mathrm{fs}$ much smaller than the pulse duration.

3 Results and discussion The transmitted intensity of the $P_{2}$ beam as a function of the transverse position of the sample in a $x y$ scan is shown in Fig. 2a. The exciting pulses are resonant with the CQDs absorption peak at $590 \mathrm{~nm}$. Regions with higher concentration of CQDs show a reduced transmission and appear as darker. The brighter area, instead, correspond to regions with maximum transmission revealing the absence of colloidal quantum dots in the volume. The corresponding FWM intensity scan of the same region is shown in Fig. 2b. The FWM vanishes at the positions where maximum transmission is observed, while is maximum when the absorption is minimum, as expected. The FWM image shows high contrast with maximum signal three orders of magnitude larger than the background. The optical sectioning of the technique is demonstrated in Fig. 2c where an $y z$ scan of the FWM intensity for a fixed $x$ position (indicated by the white dashed line of Fig. $2 b$ ) is shown. As discussed in detail in a forthcoming paper [13], we have inferred a lateral spatial resolution of $\sim 150 \mathrm{~nm}$ and an axial resolution of $\sim 590 \mathrm{~nm}$, well beyond the onephoton diffraction limit.

The dependence of the FWM intensity on the excitation intensity is shown in Fig. 3. For a total time-averaged excitation intensity (calculated from the average power over the focal area) of $P_{\text {tot }}<0.03 \mathrm{MW} / \mathrm{cm}^{2}$ (corresponding to a peak intensity of $\sim 3 \mathrm{GW} / \mathrm{cm}^{2}$ ) the FWM field amplitude is proportional to the intensity of $P_{2}$ and to the square root of the intensity of $P_{1}$, as expected from such thirdorder nonlinearity [14]. At higher excitation intensities the FWM amplitude dependence deviates from the third-order scaling and eventually completely saturates due to a saturation of the exciton density in the CQDs. Saturation of the FWM signal and of the absorption bleaching is measured for excitation powers up to $\sim 5 \mathrm{MW} / \mathrm{cm}^{2}$. Increasing the power above this level leads to an irreversible damage of the sample, possibly due to avalanche breakdown [15].

The maximum emitted FWM signal is observed for a total excitation intensity of $\sim 5 \mathrm{MW} / \mathrm{cm}^{2}$. This allows us to estimate the sensitivity limit of our FWM detection as explained in detail in [13]. Briefly, the number of quantum dots in the focal volume is estimated by measuring the intensity of the transmitted probe beam and the CQD extinction coefficient. The detected field amplitude is proportional to the number of CQDs. The measured shot noise of the reference determines a noise floor of the amplitude de- 

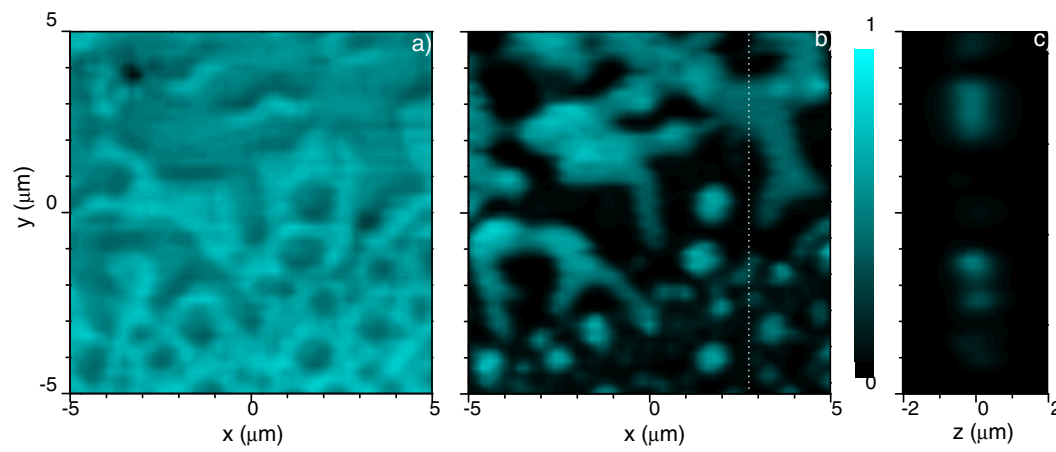

Figure 2 (a) Normalized transmitted intensity of the probe beam as a function of the transverse position of the sample. (b) Normalized fourwave mixing intensity scan of the same region as in (a). (c) $y z$ scan of the FWM intensity along a fixed $x$ position indicated by the dashed line in (b). The excitation wavelength is $590 \mathrm{~nm}$ and the excitation intensity in the focus is $13 \mathrm{~kW} / \mathrm{cm}^{2}$. The maximum FWM intensity in the color scale (red) is three orders of magnitude bigger than the non-resonant background (black). The pixel size is $0.2 \mu \mathrm{m} \times 0.2 \mu \mathrm{m}$ for the $x y$ scans and $0.2 \mu \mathrm{m} \times 0.1 \mu \mathrm{m}$ for the $y z$ scan. tection equivalent to the signal from $10 \mathrm{CQDs} / \sqrt{\mathrm{Hz}}$ in the focal volume. This sensitivity limit thus appears promising for practical applications in bio-imaging.

One of the main motivation in using colloidal nanocrystals in fluorescence imaging is their reported superior photostability compared to conventional organic dyes [7]. We have investigated the transient transmittivity of our sample, and its influence in the measured FWM, by switching the total excitation intensity from low $\left(\sim 5 \mathrm{~kW} / \mathrm{cm}^{2}\right)$ to high values $\left(\sim 43 \mathrm{~kW} / \mathrm{cm}^{2}\right)[16]$. The variation of the sample absorbance $\Delta A(t)=\log \left(I_{0} / I(t)-\log \left(I_{0} / \bar{I}\right)\right.$, where $I_{0}$ is the incident intensity, $I(t)$ is the transient measured transmitted intensity, and $\bar{I}$ is the expected transmitted intensity as if no absorption bleaching occurs ${ }^{1}$, is shown in Fig. 4 as a function of the illumination time. For $t<0 \mathrm{~s}$ the sample is excited with a total intensity of $\sim 5 \mathrm{~kW} / \mathrm{cm}^{2}$, and the ab-

${ }^{1} \bar{I}$ was measured for low excitation power where no absorption bleaching was observed and then estimated at high excitation power by assuming a linear proportionality with the incident intensity.

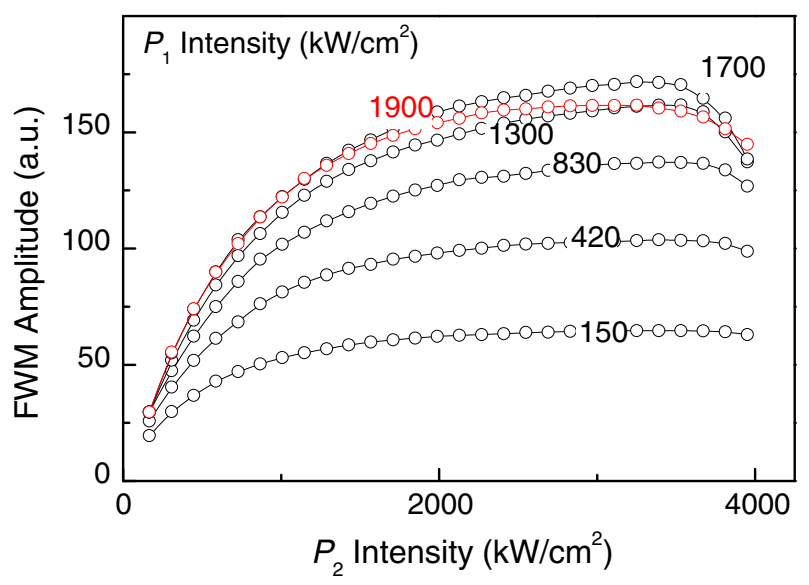

Figure 3 FWM field amplitude as function of $P_{2}$ intensity. The different curves refer to different $P_{1}$ intensities as labeled. For $P_{1}$ intensities larger than $1900 \mathrm{~kW} / \mathrm{cm}^{2}$ (red circles) a relevant absorption bleaching and a reduction of the maximum emitted FWM signal are observed. sorbance remains constant while at $t=0 \mathrm{~s}$, the total excitation intensity is instantaneously increased to $43 \mathrm{~kW} / \mathrm{cm}^{2}$. Under this high excitation the absorbance decreases with time, indicating a transient absorption bleaching, then saturates for $t \sim 10 \mathrm{~s}$. After switching the excitation density back to the low level (at $t \sim 14 \mathrm{~s}$ ) the absorbance slowly increases to the initial value as the absorption property of the sample is recovered. The dependence of the maximum $\Delta A$ on the total excitation power is shown in the inset of Fig. 4. The variation in the absorption property of the sample is negligible for low excitation intensity $\left(P_{\text {tot }}<10 \mathrm{~kW} / \mathrm{cm}^{2}\right)$, while for higher incident intensity the transmittivity of the sample increases, and then saturates for $P_{\text {tot }} \sim 100 \mathrm{~kW} / \mathrm{cm}^{2}$.

The dependence of the emitted FWM amplitude and the transmitted $P_{2}$ intensity on the illumination time are shown in Fig. 5a for an excitation intensity of $\sim 22 \mathrm{~kW} / \mathrm{cm}^{2}$ (for $t<0 \mathrm{~s} P_{\text {tot }} \sim 5 \mathrm{~kW} / \mathrm{cm}^{2}$ ). As discussed above, the transmittivity of the sample increases with increasing time. At the same time, the FWM emission is observed to decrease with a similar behavior. A

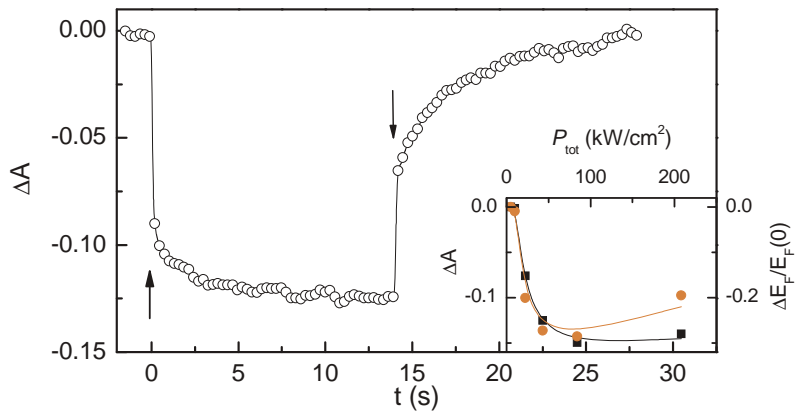

Figure 4 Variation of the sample absorbance $\Delta A$, defined in the text, as a function of the exposure time. The total excitation intensity switches from $\sim 5 \mathrm{~kW} / \mathrm{cm}^{2}$ to $43 \mathrm{~kW} / \mathrm{cm}^{2}$ at $t=0 \mathrm{~s}$ (upward arrow) and back to $\sim 5 \mathrm{~kW} / \mathrm{cm}^{2}$ at $t \sim 14 \mathrm{~s}$ (downward arrow). The time step is $\sim 0.1 \mathrm{~s}$. The inset shows the dependence of the maximum change in the absorbance (black squares) and the maximum relative variation of the FWM (red dots) on the total excitation intensity $P_{\text {tot }}$. The solid lines are a guide for the eye. 


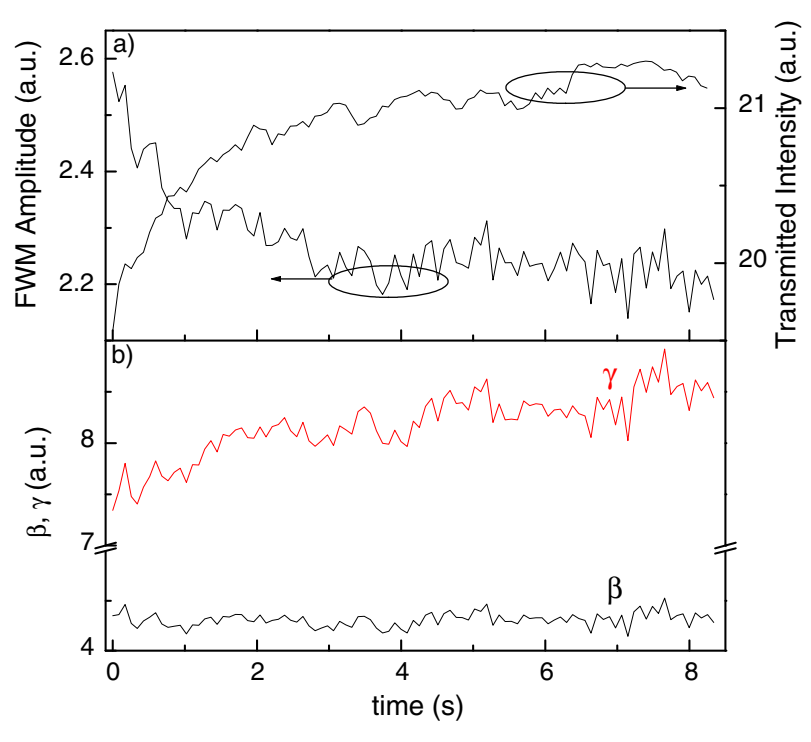

Figure 5 (a) Transmitted $P_{2}$ beam and FWM intensities for $P_{\text {tot }} \sim 22 \mathrm{~kW} / \mathrm{cm}^{2}$ (for $t<0 \mathrm{~s} P_{\text {tot }} \sim 5 \mathrm{~kW} / \mathrm{cm}^{2}$ ) as a function of the exposure time. The ratio between $P_{1}$ and $P_{2}$ power densities is 0.5 . (b) Behavior of the coefficients $\beta$ (black line) and $\gamma$ (red line), defined in the text, as a function of time. The time step is $\sim 0.1 \mathrm{~s}$.

decrease of the FWM signal under illumination can be due to effects such as a reduction of the number of optically active CQDs (possibly due to photo-induced charging), a decrease of the transition dipole moment, or a shorter dephasing time. However, the latter effect would not affect the absorption property of the CQDs, while both a reduced number of optically active nanocrystals and a decreased oscillator strength would determine a reduction in the absorptivity as well as in the FWM emission. To infer which of these two effects is more relevant we have correlated the FWM emission with the absorption coefficient as follows. If a reduced number of optically active nanocrystals is responsible for the reduced FWM emission and absorptivity, the FWM field amplitude which is proportional to the number of the absorbers (i.e. to the coherent superposition of their fields) is then directly related to the the absorption coefficient $\alpha$ by, $E_{\mathrm{F}}(t)=\beta \alpha(t)=\beta(1 / L) \ln \left(I_{0} / I(t)\right)$, where $L$ is the thickness of the sample and $\beta$ is a constant. Conversely, if the reduced FWM emission and absorptivity is due to a decreased transition dipole moment, the FWM amplitude is related to $\alpha$ by $E_{\mathrm{F}}(t)=\gamma \alpha(t)^{2}$, where $\gamma$ is a constant, since the FWM field amplitude $(\alpha)$ is proportional to the forth (second) power of the transition dipole moment.

The dependence of $\beta$ and $\gamma$ on time (calculated assuming the relationships described above) is shown in Fig. 5b. $\beta$ is found to be constant but not $\gamma$. This result shows that the prolonged excitation determines a reduction in the number of optically active CQDs rather than a transient change in their oscillator strengths. We observed that $\beta$ is not dependent on the exposure time for excitation power densities up to $\sim 100 \mathrm{~kW} / \mathrm{cm}^{2}$. The influence of the photobleaching to the FWM emission is shown in the inset of Fig 4. The relative variation of the FWM amplitude, $\left(E_{\mathrm{F}}^{\mathrm{sat}}-E_{\mathrm{F}}(0)\right) / E_{\mathrm{F}}(0)$, where $E_{\mathrm{F}}^{\text {sat }}$ is the saturation value of the FWM signal under illumination, is minor $(<30 \%)$ in the investigated excitation range and negligible $(\sim 1 \%)$ in the third-order regime.

4 Summary In summary, we have demonstrated the feasibility of a new microscopy modality based on the resonant four-wave mixing response of colloidal quantum dots. This multi-photon technique benefits from an intrinsic 3D resolution and from an interferometric detection of a coherent signal which rejects any incoherent background, for improved image contrast capabilities compared to fluorescence microscopy. The stability of FWM emission of the investigated $\mathrm{CdSe} / \mathrm{ZnS}$ CDQs was studied as a function of the excitation intensity and light exposure time. We found that a transient reversible absorption bleaching is observed at high excitation intensities and correlates with a transient change in the FWM intensity, as though a reduced number of CQDs is optically active after exposure. However this effect is minor in the investigated excitation range and negligible in the regime corresponding to a third-order behavior of the FWM nonlinearity.

\section{References}

[1] See a review on biological optical imaging in: Science 300 (2003).

[2] M. Bruchez, M. Moronne, P. Gin, S. Weiss, and A. Alivisatos, Science 281, 2013 (1998).

[3] X. Michalet, F. Pinaud, T. Lacoste, M. Dahan, M. P. Bruchez, A. Alivisatos, and S. Weiss, Single Mol. 4, 261 (2001).

[4] X. Michalet, F. F. Pinaud, L. A. Bentolila, J. M. Tsay, S. Doose, J. J. Li, G. Sundaresan, A. M. Wu, S. S. Gambhir, and S. Weiss, Science 307, 538 (2005).

[5] J. Klostranec and W. Chan, Adv. Mater. 18, 1953 (2006).

[6] A. Alivisatos, Science 271, 933 (1996).

[7] W. C. W. Chan and S. Nie, Science 281, 2016 (1998).

[8] W. Denk, J. Strickler, and W. Webb, Science 248, 73 (1990).

[9] D. Larson, W. Zipfel, R. Williams, S. Clark, M. Bruchez, F. Wise, and W. Webb, Science 300, 1434 (2003).

[10] D. Mittleman, R. Schoenlein, J. Shiang, V. Colvin, A. Alivisatos, and C. Shank, Phys. Rev. B 49, 14435 (1994).

[11] Y. Sun, P. Borri, F. Masia, and W. Langbein, unpublished.

[12] P. Borri and W. Langbein, J. Phys.: Condens. Matter 19, 295201 (2007).

[13] F. Masia, P. Borri, and W. Langbein, unpublished.

[14] J. Shah, Ultrafast Spectroscopy of Semiconductors and Semiconductor Nanostructures (Springer, Berlin, 1996).

[15] R. W. Boyd, Nonlinear Optics (Elsevier, 2003).

[16] In the experiment the ratio between $P_{1}$ and $P_{2}$ power densities is kept constant to 0.5 to have the same condition in the study of the transient FWM. 